\title{
Epitope spreading and a varying but not disease-specific GAD65 antibody response in Type I diabetes
}

\author{
P.Söhnlein ${ }^{1}$, M.Müller ${ }^{2}$, K.Syren ${ }^{2}$, U.Hartmann ${ }^{2}$, B. O. Böhm ${ }^{2}$, H.M.Meinck ${ }^{3}$, M.Knip ${ }^{4 *}$ H.K.Akerblom ${ }^{5 *}$, \\ The Childhood Diabetes in Finland Study Group*, W. Richter ${ }^{1}$ \\ ${ }^{1}$ Dept of Orthopaedic Surgery University of Heidelberg, Germany \\ ${ }^{2}$ Department of Internal Medicine 1, University of Ulm, Ulm, Germany \\ ${ }^{3}$ Department of Clinical Neurology, University of Heidelberg, Germany \\ ${ }^{4}$ Medical School University of Tampere and Deptartment of Paediatrics, Tampere University Hospital, Tampere, Finland \\ ${ }^{5}$ Hospital for Children and Adolescents, University of Helsinki, Helsinki, Finland
}

\section{Abstract}

Aims/hypothesis. The aim of this study was to analyse the conformational and linear epitope profiles of glutamic acid decarboxylase antibody (GAD65-ab)positive sera to find disease-specific epitope profiles and to study, whether GAD65-ab epitope recognition changes or spreads during the prediabetic period and, thus, can provide markers to differentiate early from later stages of progression to diabetes.

Methods. Sera from subjects before $(n=21)$, at onset $(n=44)$, or at increased risk of Type I (insulin-dependent) diabetes mellitus $(n=20)$ and from patients with stiff-man syndrome (SMS, $n=18$ ) or polyendocrine autoimmune syndrome (PAS, $n=21$ ) were analysed for conformational and linear GAD65 epitope recognition by an immunohistochemical blocking test based on human monoclonal GAD65-ab (MICA 1-10) and western blotting of a GAD65 epitope-cDNA-library.

Results. A redundant reactivity of many GAD65-ab positive sera to three major conformational (EP-1, EP-2, EP-3) and two dominant linear epitope clusters (amino acid 1-124 and 535-585) was observed in diabetes, polyendocrine autoimmune syndrome and stiff-man syndrome and no disease-specific epitopes or epitope-profiles were detected. Epitope recognition broadened with higher titres and with the vulnerability of patients to acquire additional autoimmune diseases apart from diabetes. Low GAD65-ab serum titres ( $<1200$ arbitrary units) and EP-1 recognition in the absence of EP-2 binding characterised the early immune response. Changing epitope profiles combined stable recognition of EP-1 with gain or loss of reactivity to $\mathrm{C}$-terminal epitopes during follow-up. Conclusion/interpretation. A maturing autoantibody response, which could spread from EP-1-recognition to other regions of GAD65, resulted in titre-related rather than disease-specific epitope profiles which were not sufficient to predict whether GAD65-ab positive subjects will progress to Type I diabetes, autoimmune polyendocrine syndrome or stiff-man syndrome. [Diabetologia (2000) 43: 210-217]

Keywords Glutamate decarboxylase, autoantibodies, epitope-specificity, Type I diabetes, polyendocrine autoimmune syndrome, stiff-man syndrome, prediabetes.
Received: 16 August 1999 and in revised form: 22 October 1999

Corresponding author: W. Richter, Stiftung Orthopädische Universitätsklinik Heidelberg, Schlierbacher Landstrasse 200 a, D-69118 Heidelberg, Germany

* Members of the Childhood Diabetes in Finland Study Group. For a full list of members see reference 22 .

Abbreviations: aa, Amino acid; GAD, glutcic acid decarboxylase; GAD65, the $65 \mathrm{kD}$ isoform of GAD; GAD65-ab, antibodies directed to GAD65; MICA, monoclonal islet cell antibodies; PAS, autoimmune polyendocrine syndrome; SMS, stiff-man syndrome; DIG, digoxigenin.
The $\gamma$-amino butyric acid (GABA) synthesising enzyme glutamic acid decarboxylase (GAD) is a common target of the humoral immune response in Type I (insulin-dependent) diabetes mellitus. Studies in first-degree relatives [1, 2], twins [3] and school-children $[4,5]$ have shown that GAD autoantibodies (GAD65-ab) can be present years or even decades before the clinical diagnosis of Type I diabetes. They have been used for prediction of diabetes development especially in combination with other diabetesrelated autoantibodies [6]. These GAD65-ab also ap- 
Table 1. Characterisation of study groups and their GAD65-antibody titre

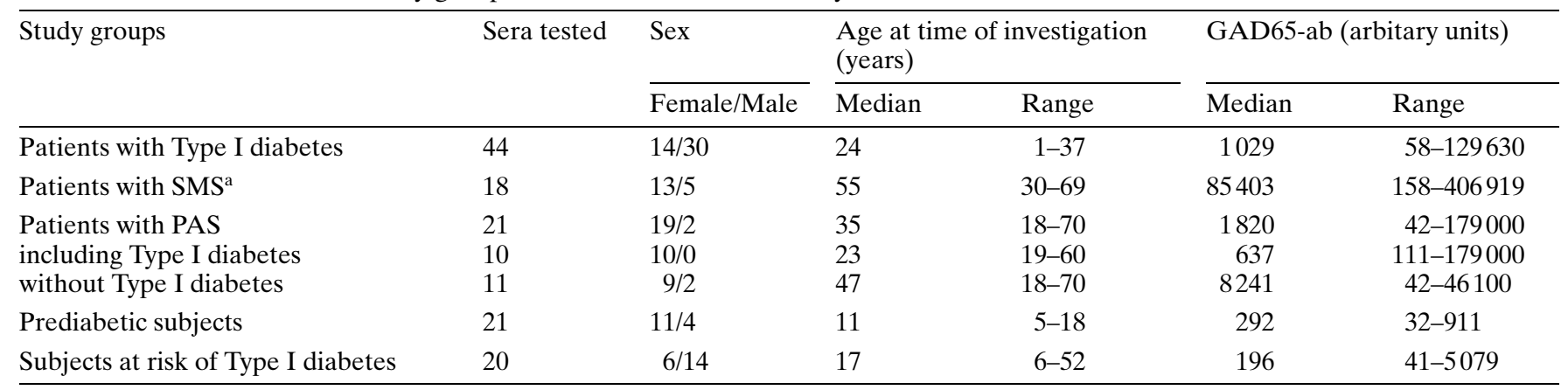

${ }^{a}$ Quantification of GAD65-ab was available for only 9 sera

pear in sera from patients with the rare neurological disorder stiff-man syndrome (SMS) $[7,8]$ and in a third group of patients, in which various autoimmune endocrinopathies coincide in diverse combinations. A minor group of these patients with autoimmune polyendocrine syndrome (PAS) develop Type I diabetes at a later stage (PAS + diabetes) whereas others do not (PAS - diabetes). It has been suggested that subclinical beta-cell inflammation, not invariably progressing to diabetes, induces GAD autoantibodies in these patients [9-11].

The smaller of the two non-allelic isoforms of GAD, GAD65, is the dominant target of autoantibodies in Type I diabetes. In contrast, SMS and PAS sera frequently recognise both GAD isoforms, GAD65 and GAD67 [12, 13]. Analyses of human monoclonal GAD65 autoantibodies (MICA 1-10) suggested that the humoral immune response to GAD65 is antigen driven and affinity maturation of antibodies occurs during progression to Type I diabetes [14]. Whether the epitope recognition of GAD65$\mathrm{ab}$ is affected by this has never been tested in sequential serum samples of GAD65-ab-positive subjects during progression to clinical manifestation of the disease. In addition, although disease-specific or risk-associated GAD65 epitope recognition had been suggested [12, 15-18], no systematic analysis, based on a large number of GAD65-ab positive sera, was available to decide whether disease-specific epitopes or epitope profiles are of diagnostic value and differentiate patients with Type I diabetes from those with PAS and SMS.

The aim of this study was to analyse the conformational and linear epitope profiles of patients with Type I diabetes, PAS and SMS to find disease-specific epitope profiles and to study whether GAD65-ab epitope-recognition changes or spreads during the prediabetic process and, thus, can provide markers to differentiate early from later stages of progression to diabetes. We established a new epitope-specific blocking test based on human monoclonal GAD65-ab (MICA) which was of optimal fine specificity to detect conformational epitopes and analysed 187 sam- ples derived from 124 GAD65-ab positive subjects for their conformational and linear GAD65 epitope profiles.

\section{Subjects and methods}

Human sera. Only GAD65-ab positive sera were included in the study (Table 1). All sera were obtained with informed consent in accordance with the principles of the declaration of Helsinki. Sera were collected from patients with Type I diabetes according to World Health Organisation criteria [19] at the clinical onset of the disease $(n=44)$ and from patients with polyendocrine autoimmune-syndrome type 2 (PAS) $(n=21)$ [20] suffering from multiple autoimmune disorders, including Hashimoto thyroiditis, Graves' disease, Addison's disease, Schmidt syndrome, Blizzard syndrome, vitiligo and Crohn's disease. Amongst patients with PAS 11 had had Type I diabetes for 2-10 years. Patients with SMS $(n=18)$ were diagnosed according to reported methods [7, 21]. Samples from 21 prediabetic subjects, i.e. subjects who subsequently progressed to Type I diabetes during longitudinal follow-up, were analysed. From them, 16 were siblings of affected children participating in the Childhood Diabetes in Finland (DiMe) study which initially comprised more than 750 unaffected siblings [22] and 5 prediabetic subjects, were from the Ulm schoolchildren study [23]. Consecutive samples from the prediabetic period were available from 10 of these subjects 18 to 96 months before the diagnosis of clinical disease. We also studied samples from 20 subjects at increased risk of Type I diabetes comprising 13 islet cell antibody-positive and GAD65-ab-positive first-degree relatives of affected patients and 7 schoolchildren testing positive for GAD65-ab (Table 1).

Quantification of GAD65-ab in sera. The radioimmunoassay for detection of GAD65-ab in sera had been described previously [24]. Briefly, in vitro transcription and translation of cDNA encoding for full-length human GAD65 (Dr. Å. Lernmark, Seattle, Washington) was carried out in the presence of ${ }^{35} \mathrm{~S}$-methionine using an SP6 coupled reticulocyte lysate system (Promega, Madison, Wis., USA). Radioactive GAD65 was separated from non-bound ${ }^{35} \mathrm{~S}$-methionine by gel filtration. Serum $(2.5 \mu \mathrm{l})$ or standard antibody MICA 3 was incubated with $20000 \mathrm{cpm}$ radioactive GAD65 in round bottom microtitre wells at $4{ }^{\circ} \mathrm{C}$ overnight. Protein A Sepharose $(1 \mu \mathrm{g}$, Pharmacia, Uppsala, Sweden) was added for $1 \mathrm{~h}$ before immune complexes were separated from unbound GAD65 by washing in membrane-bottomed microtitre wells $(0.6 \mu \mathrm{m}$ pore 
Table 2. Blocking of DIG-labelled MICA by unlabelled MICA 1-10 in the immunohistological blocking test

\begin{tabular}{|c|c|c|c|c|c|c|c|c|}
\hline \multirow{2}{*}{$\begin{array}{l}\text { Epitope } \\
\text { Cluster }\end{array}$} & \multirow{2}{*}{$\begin{array}{l}\text { Unlabelled } \\
\text { MICA }\end{array}$} & \multicolumn{7}{|c|}{ Digoxigenin-labelled MICA } \\
\hline & & 1 & 2 & 3 & 4 & 7 & 8 & 10 \\
\hline \multirow[t]{2}{*}{ EP-1 } & MICA 4 & - & - & - & blocked & - & - & blocked \\
\hline & MICA 10 & - & - & - & blocked & - & - & blocked \\
\hline \multirow[t]{2}{*}{$\mathrm{EP}-2$} & MICA 1 & blocked & - & blocked & - & - & - & - \\
\hline & MICA 3 & blocked & - & blocked & - & - & - & - \\
\hline \multirow{3}{*}{ EP-3 } & MICA 7 & - & blocked & - & - & blocked & blocked & - \\
\hline & MICA 8 & - & - & - & - & blocked & blocked & - \\
\hline & MICA 9 & - & - & - & - & - & blocked & - \\
\hline
\end{tabular}

size, Millipore, multiscreen system, Eschborn, Germany) and the bound radioactivity was determined in a beta-counter (Wallac, Turku, Finland). The radioactivity precipitated by $0.125 \mu \mathrm{g}$ MICA 3 was defined as 100 arbitrary units (detection limit 30 arbitrary units) and results of serum samples expressed in units deduced from the standard curve. In the first combined autoantibody workshop (1995) the GAD65-ab assay had a sensitivity of $70.4 \%$ and a specificity of $100 \%$. Due to the limited serum sample only 9 of the 18 SMS sera could be quantified.

Epitope-specific blocking test. The human monoclonal antibodies MICA 1-10, isolated from three patients newly diagnosed with Type I diabetes, were described elsewhere [25-27]. We purified MICA 1, 2, 3, 4, 7, 8 and 10 by Protein A Sepharose affinity chromatography as described [28] and labelled them with digoxigenin (DIG) using a DIG protein labelling kit (Boehringer Mannheim, Mannheim, Germany). The low antibody productivity of MICA 5, 6, and 9 cell lines did not allow DIG labelling of these antibodies; they were however included as primary antibodies in the blocking tests.

Unfixed cryostat sections of human pancreas were incubated $\left(1 \mathrm{~h}, 20^{\circ} \mathrm{C}\right)$ with $10 \mu \mathrm{l}$ serum or $30 \mu \mathrm{l} \mathrm{MICA}$ supernatant $(1 \mu \mathrm{g} \mathrm{ab} / \mathrm{ml})$ as primary antibodies. The cryostat sections were washed $(15 \mathrm{~min}$, PBS: $137 \mathrm{mmol} / \mathrm{l} \mathrm{NaCl}, 2 \mathrm{mmol} / \mathrm{l} \mathrm{KCl}$, $6 \mathrm{mmol} / \mathrm{l} \quad \mathrm{Na}_{2} \mathrm{HPO}_{4}, 1.4 \mathrm{mmol} / \mathrm{l} \mathrm{KH}_{2} \mathrm{PO}_{4} \mathrm{pH}$ 7.2) and the DIG-labelled MICA $(30 \mu \mathrm{l})$ were applied as secondary antibody at end-point titre (see below) $\left(1 \mathrm{~h}, 20^{\circ} \mathrm{C}\right)$. After a washing step (10 min PBS), binding of DIG labelled MICA was detected by the third antibody, a peroxidase-conjugated goat anti-DIG antiserum (30 $\mu \mathrm{l}, 1 / 300$ dilution in $100 \mathrm{mmol} / \mathrm{l} \mathrm{TRIS} /$ $\mathrm{HCl}, \mathrm{pH}$ 7.5; Boehringer Mannheim, Mannheim, Germany). Sections were washed twice in $\operatorname{PBS}\left(5 \mathrm{~min}, 4^{\circ} \mathrm{C}\right)$, fixed for $3 \mathrm{~min}$ in $50 \mathrm{mmol} / \mathrm{l}$ sodium acetate $(\mathrm{pH} \mathrm{5})$ and stained with $1 \mathrm{mmol} / 1$ 3-amino-9-ethyl-carbazole/sodiumacetate ( $\mathrm{pH} 5)$, including $0.07 \%(\mathrm{v} / \mathrm{v})$ hydrogen peroxide. The sections were washed with water for $5 \mathrm{~min}$ and counterstained with hemalum solution (Merck, Darmstadt, Germany). The end-point titre was the highest dilution of each MICA-DIG at which the GAD65-ab-negative normal serum did allow staining of MICA-DIG whereas preincubated unlabelled MICA $(1 \mu \mathrm{g} /$ $\mathrm{ml}$ ) completely blocked the binding of MICA-DIG. This dilution was 1:300 for MICA 2-DIG, 1:8000 for MICA 3-DIG, 1:10000 for MICA 4-DIG, 1:2000 for MICA 7-DIG and 1:500 for MICA 8-DIG. Epitope specific binding of GAD65-ab positive sera was analysed using these optimised amounts of DIGlabelled MICA as secondary antibody. Fully or weakly stained islets indicated that the MICA epitope had not been covered by GAD65-ab in the serum. Unstained islets showed that the corresponding MICA epitope was blocked by GAD65-ab from serum which recognised either the same epitope as the DIG-labelled MICA or a closely related epitope.

Western blot analysis. Full length human GAD65 and fragments obtained from an epitope cDNA library of GAD65 were expressed as fusion proteins with glutathione S-transferase in E. coli according to a reported method [29]. Proteins were subjected to western blotting as described [29]. Briefly, recombinant GAD65 and GAD65-fragment fusion proteins were separated by SDS-PAGE according to [30] and transferred to nitrocellulose (Hybond $\mathrm{C}$ super, Amersham Buchler, Braunschweig, Germany) [31]. The immobilised proteins were incubated for $2 \mathrm{~h}$ with diluted sera (SMS-sera 1:500, all other sera 1:100) which had been pre-absorbed with $0.1 \mathrm{mg} / \mathrm{ml}$ crude E. coli extract to prevent background staining by $E$. coli-specific antibodies. We used MICA 2 cell culture supernatant $(1.4 \mu \mathrm{g} / \mathrm{ml})$ or reference sera as positive controls. Bound antibodies were visualised by alkaline phospatase-conjugated goat anti human antibodies (Dianova, Hamburg, Germany) and stained with nitro-blue-tetrazolium/5 bromo-4-chloro-3indolylphosphate.

Statistical analysis. Association between diagnosis and epitope clusters was analysed by explorative testing using Fisher's exact test. Group comparisons of GAD65 titres were either done by the Kruskal-Wallis H Test or the Mann-Whitney U Test, as appropriate. Two-tailed $P$ values of 0.05 or less were considered to indicate statistical significance.

\section{Results}

Identification of three independent conformational epitope clusters EP-1-3. To determine how many epitopes can be distinguished by the MICA based epitope-specific test, MICA 1-10 were analysed for their capacity to block each other's binding to GAD65 in this test. The MICA 4, 6, and 10 competed for binding to GAD65 (Table 2) and thus, bound sterically closely related epitopes within an epitope cluster which we called EP-1. A distinct but identical blocking pattern was found for MICA 1 and 3 (Table 2) defining a further epitope-cluster called EP-2. The MICA 2, 5, 7, 8, and 9 competed for binding to GAD65, addressing a third epitope cluster (EP-3). The blocking pattern of MICA 5 was identical to MICA 7 and a unique but 


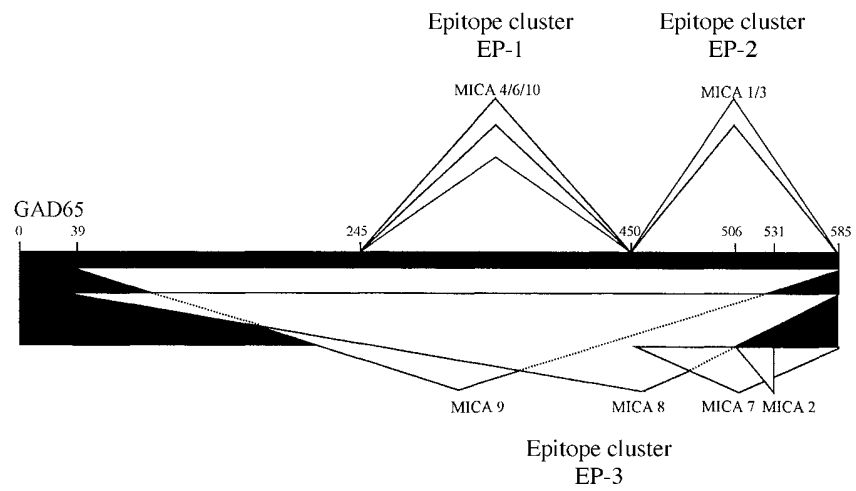

Fig. 1. MICA 1-10 compete for binding to three major GAD65 epitope clusters (EP-1 - EP-3). Linearised plot of GAD65 with amino acids given for domain boundaries identified by analysis of GAD65 deletion mutants and GAD65/ GAD67 chimeric molecules with the MICA [27]. The epitope recognition of each MICA is indicated by a triangle spanning the GAD65 region required for binding of the antibody. Overlapping triangles indicate competition for binding to GAD65 and non-interacting triangles represent MICAs which can bind simultaneously to GAD65. A maximum of four MICA, like MICA 4, 3, 7, and 9 can bind simultaneously to GAD65 in its in vivo localisation in beta cells

Fig. 2. Prevalence of GAD65 antibodies recognising MICA epitopes. Sera from patients with stiff-man syndrome (SMS), autoimmune polyendocrine syndrome with (PAS + diabetes) or without Type I diabetes (PAS - diabetes) from patients with Type I diabetes, as well as from prediabetic subjects or subjects at high risk were analysed in the epitope-specific histochemical blocking test. Conformational epitopes recognised by GAD65-ab-positive sera were analysed with MICA 4-DIG for detection of EP-1 (black bars) and MICA 3-DIG for EP-2 specificities (white bars). MICA 2-DIG, 7-DIG, and 8-DIG were used to distinguish the four distinct conformational epitopes in EP-3 (hatched bars). Because there could have been several GAD65-ab specificities in polyclonal sera, the epitope patterns in EP-3 had to be discriminated subtractively as EP-3 (MICA 7/5 $\pm 2 \pm 8 \pm 9$ dark hatched pattern), EP-3 minus 2/5/7 (MICA 8, 9; medium hatched pattern) and EP-3 minus 2/5/7/8 (only MICA 9; light hatched pattern). Median titre and 25 or $75 \%$ quartiles are indicated for each study group not independent blocking pattern was found for MICA 2, MICA 8 and MICA 9, indicating that their epitopes were sterically related to each other. Blocking was identical irrespective which of the two MICA was used for blocking or for detection of the epitope except for MICA 2/8. This showed that binding affinity [14] had no major effect on displacement of antibodies in our test. According to previous data [27], EP-1 resides in the middle region of GAD65 between amino acid (aa) 245 and 450 whereas EP-2 and EP-3 lie at the C-terminus from aa 450 to 585 (Fig. 1). Accordingly, from nine distinct epitopes defined by the ten MICA [32], six can be distinguished by our epitope-specific blocking test. Competition for binding to only three independent epitope clusters shows, however, that in spite of a highly diverse fine specificity, GAD65-ab target only a few immunodominant domains in the native molecule.

Common conformational epitope recognition in Type I diabetes, SMS and PAS. Sera positive for GAD65ab from patients with newly diagnosed Type I diabetes, with PAS + diabetes or PAS - diabetes and from patients with SMS (Table 1) showed GAD65-ab to the three epitope clusters in high frequency and bound to all six distinguishable MICA epitopes (Fig.2). Antibodies to all three epitope clusters were detected in 44 of the 83 sera (53\%); 22 sera (27\%, 20 Type I diabetes, 2 PAS - diabetes sera) were negative for all three epitope clusters. Sera positive for antibodies to EP-1, or EP-2 or EP-3 or any combination of them had higher titres of GAD65 antibodies (median titre $19889, p<0.001$ ) than sera negative for EP-1, EP-2 and EP-3 (median titre 304) indicating a relation between the frequency of EP-1-3 recognition and titre, which was also obvious within the study groups. Parallel to a lower GAD65-ab titre (Fig.2), the frequency of EP-1, EP-2 and EP-3-reactive sera was significantly lower in patients with Type I diabetes compared with SMS patients $(p<0.007)$ but the differences did not reach significance to the smaller PAS + diabetes and PAS - diabetes groups (Fig.2). A large subgroup of patients with Type I diabetes had an overlapping titre-range to subjects with

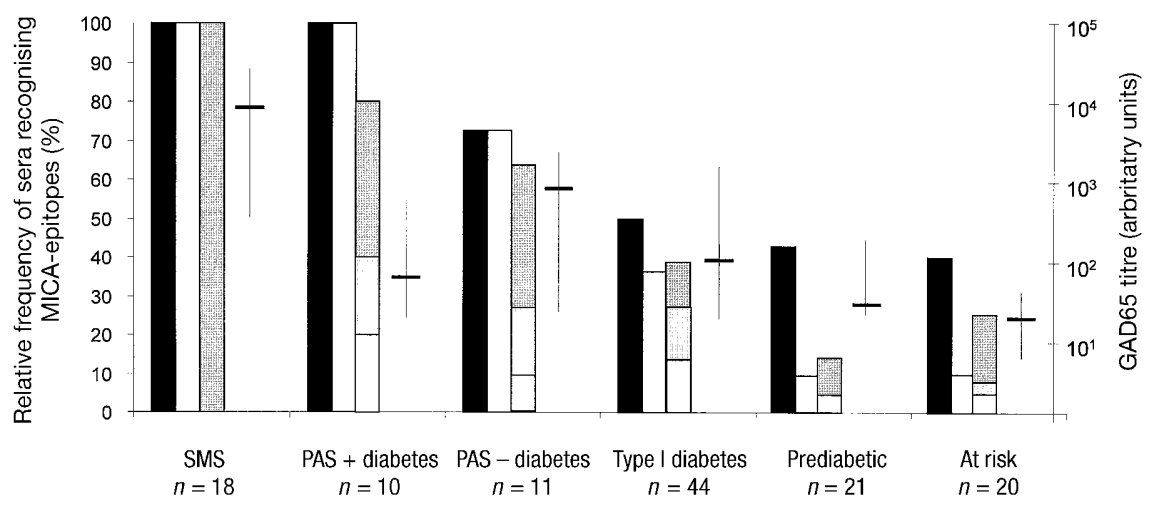



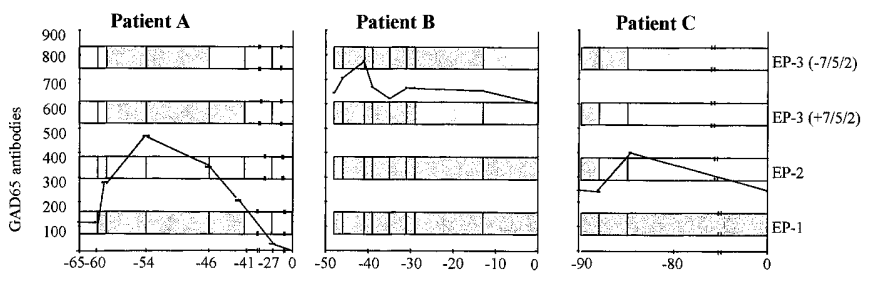

Months before clinical onset of diabetes

Fig.3. Variable epitope reactivity in prediabetic subjects during progression to overt Type I diabetes. Semiquantitative analysis of epitope recognition by the histochemical blocking tests in three prediabetic subjects $(\mathrm{A}-\mathrm{C})$ before the clinical onset of Type I diabetes. White bars indicate the absence of GAD65 antibodies to the respective epitope cluster, light hatched bars indicate weak reactivity (partial blocking) and dark hatched bars strong reactivity (complete blocking) with epitope clusters EP-1 to EP-3. The GAD65 autoantibody titre of the serum samples is indicated in arbitrary units

PAS + diabetes allowing titre-matched subgroups to be analysed for their epitope profiles.

The titre-matched group of PAS + diabetes patients recognised all MICA-defined epitope clusters more frequently than sera from patients with diabetes only (PAS + diabetes: $n=9$, median titre $=387$ : EP$1=9(100 \%), \mathrm{EP}-2=9(100 \%), \mathrm{EP}-3=7(77 \%) ;$ Type I diabetes: $n=29$, median titre $=397: \mathrm{EP}-1=8$ $(28 \%), \mathrm{EP}-2=4(14 \%), \mathrm{EP}-3=3(10 \%$; all comparisons: $p<0.001)$. Thus, like higher GAD65-ab titres, the occurrence of other autoimmune diseases apart from diabetes increased the prevalence of antibodies directed to EP-1, EP-2 and EP-3 significantly. No diabetes-specific epitope profiles could, therefore, be defined because the number and combination of EPreactive antibodies varied greatly with titre and associated disorders apart from diabetes.

Dominant and early immune reactivity is directed to EP-1. Frequently, GAD65-ab have been observed long before clinical diagnosis of Type I diabetes [1-5]. In GAD65-ab positive prediabetic subjects and subjects at risk of developing diabetes we found lower median GAD65-ab titres like in diabetic patients at clinical onset of the disease (Table 1). In spite of this, a high frequency of antibodies to EP-1 was observed whereas the reactivity to EP-2 and to part of EP-3 was significantly reduced (Fig. 2) (EP-2: prediabetic subjects: $p=0.022$; subjects at risk: $p=0.020$; Cterminal part of EP-3 (MICA $8+9$ ) (light grey in Fig. 2): prediabetic subjects, $p=0.06$ : subjects at risk; $p=0.05)$. In titre-matched subgroups of prediabetic subjects or at-risk subjects the recognition of EP-1 in the absence of EP-2 binding was striking compared with subjects newly diagnosed with diabetes. Considering only sera positive for any EP-epitopes seven of nine prediabetic subjects $(p=0.029)$, six of seven atrisk subjects $(p=0.041)$ compared with only one of seven newly diagnosed diabetic subjects showed this epitope pattern. Thus, EP-1 positivity combined with EP-2 negativity at serum titres below 1200 arbitrary units was characteristic for early stages of the GAD65-ab response. This indicated, that the middle region of GAD65 (EP-1) attracted an early and immunodominant response while $\mathrm{C}$-terminal parts of GAD65 could be recognised later on or at higher titres, a hypothesis which could be confirmed with follow-up sera from GAD65-ab positive subjects before onset of diabetes.

Variable epitope recognition during progression to Type I diabetes. In five of ten available (50\%) prediabetic subjects, GAD65-ab directed to MICA-defined epitopes were identified during a follow-up of 18-96 months before clinical onset of diabetes. Over time the epitope profile was constant in two and variable in three subjects. Constant epitope recognition was directed to EP-1 alone or in combination with EP-2 and EP-3 over 43 or 45 months before clinical onset, respectively. Changing epitope profiles included gain (Fig. 3A) and loss of epitope recognition (Fig. 3A-C) to the C-terminal epitope clusters (EP-2 and EP-3) but only in one case (Fig. 3A) did the GAD65-ab titre change accordingly. Among seven high-risk subjects (GAD65-ab positive school children), who had not progressed to overt Type I diabetes, three $(43 \%)$ showed reactivity towards MICAdefined epitopes in the first sample. In two of them antibodies to EP-1 were present in the first available sample (GAD65-ab titre 2927 and 574 arbitrary units) and one serum (328 arbitrary units) was negative for MICA-defined epitopes. Follow-up samples taken 8 years later showed epitope spreading from EP-1 to EP-2 and EP-3 in one subject and from negative to EP-1 and EP-2 in another. A third subject had lost her EP-1-recognition. In all subjects positive for MICA-defined epitopes at any time $(n=8)$, EP-1 was always the first or among the first epitopes recognised by GAD65-ab confirming our hypothesis, that this region is an early immunodominant epitope region recognised even at low titres of GAD65-ab. Changing epitope profiles always combined a stable reactivity to this dominant epitope cluster with appearing or disappearing reactivity to the C-terminal epitopes.

The major linear epitope clusters map to both ends of $G A D 65$. To complete the epitope profiles of sera from the different study groups, the prevalence of antibodies directed to linear GAD65 epitopes was analysed by western blot on recombinant human GAD65. Linear epitopes were detected in $83 \%(15 / 18)$ of the SMS sera and $60 \%(6 / 10)$ of the PAS + diabetes sera, in contrast to only $2 / 11(18 \%$ ) sera of PAS - diabetes and $7 \%$ (3/44) sera of patients with Type I diabetes. More than one linear epitope was bound by seven SMS and two PAS + diabetes sera. The titre of 

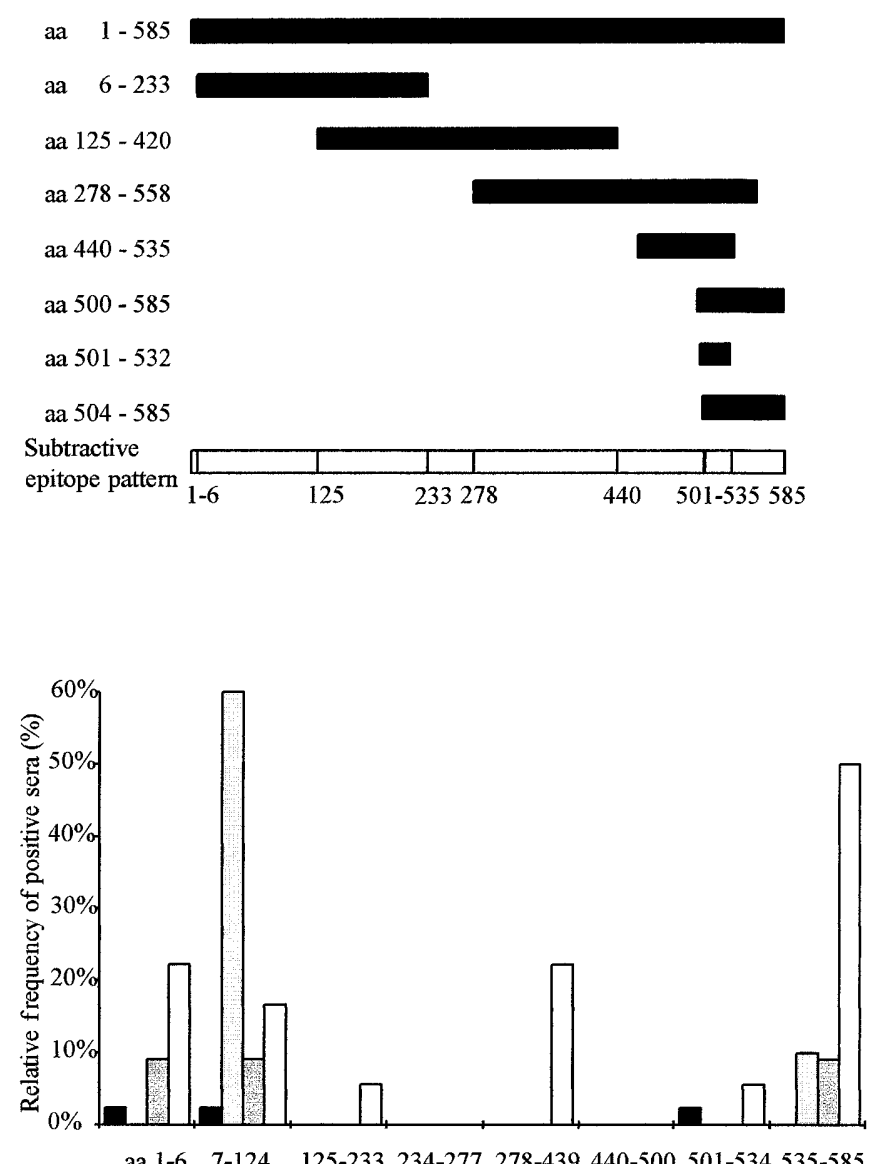

Fig. 4A, B. Linear epitope recognition of GAD65-ab-positive sera. A Linear epitopes in GAD65 were mapped using fragments from a human GAD65 cDNA epitope library. GAD65 fragments were expressed as fusion proteins with glutathione $\mathrm{S}$ transferase in $E$. coli and reactivity of the sera with all fragments was analysed by western blotting. Location of the linear epitopes was determined according to the subtractive epitope pattern given underneath. The first and the last amino acid (aa) of GAD65 expressed in the fusion protein is indicated. B Peak recognition of linear epitopes occurs in the N-terminal 124 and the C-terminal 50 aa. Linear epitope recognition of sera from patients with Type I diabetes ( $\square)$, PAS with $(\square)$ or without Type I diabetes ( $\square$ ), and from patients with SMS syndrome $(\square)$ was analysed by western blotting on the fragments shown in $\mathbf{A}$. The relative frequency of western blot positive sera reacting with individual epitope regions was determined for each study group

western blot positive sera was significantly higher (median 23.611 arbitrary units, $n=17, p=0.007$ ) than the GAD65-ab titre of western blot negative sera (median 1.008 arbitrary units, $n=57$ ). Fragments of an epitope cDNA library of human GAD65 were used to map the location of these linear epitopes (Fig.4A). The major region of GAD65 between aa 125 and 532 turned out to be almost unreactive, because it was recognised by only a few SMS sera (Fig. 4B). In all study groups dominant linear epitope recognition was directed to the N-terminal 124 aa $(17 / 26$ sera, $65 \%)$ and the C-terminal 50 aa (12/26 sera $46 \%$ ), respectively. Most striking was the frequent recognition of a linear epitope within aa 6-124 by sera of all study groups and especially by the PAS + diabetes group (Fig. 4B). Recognition of linear epitopes was more frequent in sera with higher GAD65-ab titres, an observation which had also been made for the conformational epitopes. Again, the PAS + diabetes study group was different having a high frequency of linear epitope recognition $(60 \%)$ in spite of a relatively low median GAD65-ab titre.

\section{Discussion}

Previous epitope studies on GAD65-ab in sera from patients with Type I diabetes and on human monoclonal GAD65-ab had been limited to GAD65-specific samples because the tests applied were based on analysis of recombinantly expressed chimeric GAD65/GAD67 molecules for epitope detection $[33,17]$. Disease-specific GAD65 epitope recognition could not be studied, because PAS, SMS and about $10 \%$ of diabetes sera recognise both isoforms of GAD $[9,12,13]$ and were, thus, not informative in these assays. We here established a new GAD65 epitope-specific test applicable to GAD65/GAD67 cross-reactive sera which combined the optimal fine specificity of human monoclonal GAD65-ab with the detection of native GAD65 epitopes on cryostat sections of human pancreas. Although the detection antibodies MICA 1-10 were directed to nine different GAD65 epitopes [32], they competed for binding to only three major conformational epitope clusters; one located in the middle (EP-1, aa 245-450) and two in the C-terminal region (EP-2, EP-3, aa 450-585) of GAD65. This facilitated conformational epitope analysis of polyclonal sera, reducing a highly complex molecular epitope pattern to recognition of three dominant epitope regions.

Although conformational epitope clusters EP-1-3 were a common target of GAD65-ab in diabetes $(50 \%)$, PAS - diabetes $(71 \%)$, PAS + diabetes $(90 \%)$, and in SMS (100\%) no disease-specific epitopes or epitope profiles became evident. Parallel to higher antibody titres, a broader epitope recognition was observed within and between study groups with one exception; in spite of relatively low GAD65-ab titres patients suffering from additional autoimmune diseases apart from diabetes (PAS + diabetes) showed a higher frequency of antibodies towards the conformational and linear epitopes we analysed. Especially linear epitope-recognition, which had previously been reported to be titre related [34], was surprisingly high in sera from PAS + diabetes patients. The confined location of linear epitopes at the N-terminal and C-terminal end of GAD65 which had been claimed to be SMS-specific [12, 35], was here 
seen for western blot positive sera from all study groups. We failed to detect disease-specific epitope profiles in our study and identified high GAD65-ab titres and the vulnerability of patients to acquire additional autoimmune diseases apart from diabetes as promoting variables for the spreading of the autoantibody response in GAD65. This suggests that common molecular mechanisms lead to the formation of GAD65-ab irrespective of whether islets, neurons or no apparent targets were affected by the disease.

In animal models prevention of diabetes was more effective when immune intervention was applied early, before the commencement of destructive immunity occurred [36]. Early phases of the immune response to GAD65 could, therefore, be clinically relevant not only for diagnostic purposes but also for therapeutic approaches aimed at disease prevention. One characteristic for the early GAD65-ab response was the presence of low antibody titres because $100 \%$ of the prediabetic subjects and $90 \%$ of subjects at increased risk had GAD65-ab titres below 1200 arbitrary units compared with $52 \%$ at onset of diabetes. This confirmed previous data reporting lower titres in GAD65-ab positive healthy subjects compared with patients at clinical onset [33]. Many diabetes sera and sera from prediabetic subjects with GAD65-ab titres below 1200 arbitrary units did not bind either EP-1, EP-2 or EP-3. Therefore, epitope clusters independent of EP-1-3 could exist. Alternatively, EP-1-3reactive antibodies, each below the detection level of the corresponding test, and probably in combination with GAD65-ab targeting other epitope clusters, add up to an amount of GAD65-ab easily detected by radioimmunoassay. When most of the GAD65-ab in the serum sample targeted only one epitope cluster, as seen in two EP-1 or EP-3 positive sera with very low GAD65-ab titres (42 and 60 arbitrary units), the sensitivity of the radioimmunoassay (30 arbitrary units) was, however, quite close to that of the epitope-specific test.

When we looked for early epitope patterns in follow-up samples during progression to overt disease, three lines of evidence suggested that the early and dominant GAD65-ab reactivity was directed to the middle region of GAD65: firstly, antibodies to EP-1 were the most prominent antibodies in prediabetic subjects and healthy at-risk subjects; secondly, titrematched early study groups showed an increased prevalence of EP-1 antibodies compared with sera at onset of Type I diabetes and thirdly, all follow-up samples showed EP-1 antibodies as the first or amongst the first GAD65-ab. In contrast, reactivity to C-terminal epitope areas was significantly lower than EP-1 reactivity before diabetes onset and could be gained or lost during the follow-up over years either parallel to changes in serum titre or independent from them. Thus a second characteristic of the early immune response was the recognition of EP-1 in the absence of EP-2 binding in these early study groups which, in combination with the lower titre, could serve as a marker to discriminate earlier from later stages of the GAD65-ab response. Because no common patterns of epitope spreading were observed, some subjects progressed to diabetes regardless of changes in the epitope repertoire and no disease-specific epitope profiles evolved, epitope-based followup tests of GAD65-ab will not be sufficient to predict whether GAD65-ab positive healthy subjects will progress to diabetes, SMS, PAS or any of these diseases. A comined analysis of GAD65-ab, additional organspecific autoantibodies and their epitope recognition during follow-up may, thus, be required to improve risk assessment for development of diabetes, SMS or PAS.

Acknowledgements. This study was supported by grants from the Human Frontier Science Programme, the German Research Organisation (DFG Ri 707/1-3) and the Volkswagen Foundation to W. Richter. We thank J. Endl for digoxigenin labelled MICA 4, U. Scheidt for technical assistance, and D. Brocai for statistical analyses.

\section{References}

1. Riley WJ, Maclaren NK, Krischer et al. (1990) A prospective study of the development of diabetes in relatives of patients with insulin-dependent diabetes. N Engl J Med 323: 1167-1172

2. Bingley PJ, Christie MR, Bonifacio E et al. (1994) Combined analysis of autoantibodies improves prediction of IDDM in islet cell antibody-positive relatives. Diabetes 43: 1304-1310

3. Verge CF, Gianani R, Yu L et al. GS (1995) Late progression to diabetes and evidence for chronic beta-cell autoimmunity in identical twins of patients with type 1 diabetes. Diabetes 44: 1176-1179

4. Landin-Olsson M, Karlsson A, Dahlquist G, Blom L, Lernmark A, Sundkvist G (1989) Islet cell and other organ-specific autoantibodies in all children developing Type I (insulin-dependent) diabetes mellitus in Sweden during one year and in matched control children. Diabetologia 32: 387-395

5. Schatz D, Krischer J, Horne G et al. (1994) Islet cell antibodies predict insulin-dependent diabetes in United States school age children as powerful as in unaffected relatives. J Clin Invest 93: 2403-2407

6. Aanstoot HJ, Kang SM, Kim J et al. (1996) Identification and characterization of glima 38 a glycosylated islet cell membrane antigen which together with GAD65 and IA2 marks the early phases of autoimmune response in type 1 diabetes. J Clin Invest 97: 2772-2783

7. Meinck HM, Ricker K, Huelser PJ, Schmid E, Pfeiffer J, Solimena M (1994) Stiff man syndrome: clinical and laboratory findings in eight patients. J Neurol 241: 157-166

8. Solimena M, Folli F, Aparisi R, Pozza G, DeCamilli P (1990) Autoantibodies to GABA-ergic neurons and pancreatic beta cells in stiff man syndrome. N Engl J Med 322: 1555-1560

9. Tuomi T, Bjorses P, Falorni A et al. (1996) Antibodies to glutamic acid decarboxylase and insulin-dependent diabetes in patients with autoimmune polyendocrine syndrome type I. J Clin Endocrinol Metab 81: 1488-1494 
10. Betterle C, Presotto F, Magrin L et al. (1994) The natural history of pre-Type I (insulin-dependent) diabetes mellitus in patients with autoimmune endocrine diseases. Diabetologia 37: 95-103

11. Bosi E, Becker F, Bonifacio E et al. (1991) Progression to type 1 diabetes in autoimmune endocrine patients with islet cell antibodies. Diabetes 40: 977-984

12. Butler MH, Solimena M, Dirkx R Jr, Hayday A, De Camilli P (1993) Identification of a dominant epitope of glutamic acid decarboxylase (GAD-65) recognized by autoantibodies in stiff-man syndrome. J Exp Med 178: 2097-2106

13. Seissler J, Bieg S, Yassin N et al. (1994) Association between antibodies to the MR 67000 isoform of glutamate decarboxylase (GAD) and Type 1 (insulin-dependent) diabetes mellitus with coexisting autoimmune polyendocrine syndrome type II. Autoimmunity 19: 231-238

14. Richter W, Jury KM, Loeffler D, Manfras BJ, Eiermann $\mathrm{TH}$, Boehm BO (1995) Immunoglobulin variable gene analysis of human autoantibodies reveals antigen-driven immune response to glutamate decarboxylase in type 1 diabetes mellitus. Eur J Immunol 25: 1703-1712

15. Baekkeskov S, Aanstoot HJ, Christgau S et al. (1990) Identification of the $64 \mathrm{~K}$ autoantigen in insulin-dependent diabetes as the GABA-synthesizing enzyme glutamic acid decarboxylase. Nature 347: 151-156

16. Ujihara N, Daw R, Gianani R, Boel E, Yu L, Powers AC (1994) Identification of glutamic acid decarboxylase autoantibody heterogeneity and epitope regions in type 1 diabetes. Diabetes 43 968-975

17. Daw K, Powers AC (1995) Two distinct glutamic acid decarboxylase auto-antibody specificities in IDDM target different epitopes. Diabetes 44: 216-220

18. Daw K, Ujihara N, Atkinson M, Powers AC (1996) Glutamic acid decarboxylase autoantibodies in Stiff-Man syndrome and insulin-dependent diabetes mellitus exhibit similarities and differences in epitope recognition. J Immunol 156: 818-825

19. World Health Organization Study Group on Diabetes Mellitus (1985) Technical report series No 727, WHO, Geneva

20. Skordis N, Maclaren N (1988) Immunogenetics of autoimmune polyglandular syndromes In: NR Farid (ed) Immunogenetics of endocrine disorders. Liss AR, New York, pp 373-399

21. Gordon EE, Januszko DM, Kaufman L (1967) A critical survey of stiff-man syndrome. Am J Med 42: 582-599

22. Kulmala P, Savola K, Peterson JS et al. (1998) Prediction of insulin-dependent diabetes mellitus in siblings of children with diabetes: a population based study. J Clin Invest 101: 327-336

23. Boehm BO, Manfras B, Seissler J et al. (1991) Epidemiology and immunogenetic background of islet cell antibody positive nondiabetic schoolchildren: the Ulm-Frankfurt population study. Diabetes 40: 1435-1459
24. Wiest-Ladenburger UW, Hartmann R, Hartmann U, Berling K, Boehm BO, Richter W (1997) Combined analysis and single step detection of GAD65 and IA2 autoantibodies in IDDM can replace the histochemical islet cell antibody test. Diabetes 46: 565-571

25. Richter W, Endl J, Eiermann TH et al. (1992) Human monoclonal islet cell antibodies from a patient with insulin-dependent diabetes mellitus reveal glutamate decarboxylase as the target antigen. Proc Natl Acad Sci USA 89: 8467-8471

26. Richter W, Mertens T, Schoel et al. (1994) Sequence homology of the diabetes-associated autoantigen glutamate decarboxylase with coxsackie $\mathrm{B} 4-2 \mathrm{C}$ protein and heat shock protein 60 mediates no molecular mimicry of autoantibodies. J Exp Med 180: 721-726

27. Syren K, Lindsay L, Stoehrer B et al. (1996) Immune reactivity of diabetes-associated human monoclonal autoantibodies defines multiple epitopes and detects two domain boundaries in glutamate decarboxylase. J Immunol 157: 5208-5214

28. Ey PL, Prowse SJ, Jenkin CR (1978) Isolation of pure IgG1 IgG2 a and IgG2 b immunoglobulins from mouse serum using protein A-Sepharose. Biochemistry 15: 429-436

29. Mauch L, Seissler J, Haubruck H et al. (1993) Baculovirusmediated expression of human $65 \mathrm{kDa}$ and $67 \mathrm{kDa}$ glutamic acid decarboxylase in Sf9 insect cells and their relevance in diagnosis of insulin-dependent diabetes mellitus. J Biochem 113: 699-704

30. Laemmli UK (1970) Cleavage of structural proteins during the assembly of the head of bacteriophage T4. Nature 227: 680-685

31. Towbin H, Staehlin T, Gordon J (1979) Electrophoretic transfer of proteins from polyacrylamide gels to nitrocellulose sheets; procedure and some applications. Proc Natl Acad Sci USA 79: 4350-4354

32. Schwartz H, Chandonia JM, Kash S et al. (1999) High resolution autoreactive epitope mapping and structural modeling of human glutamic acid decarboxylase (GAD65). Mol Cell Biol 287: 983-999

33. Falorni A, Ackefors M, Carlberg C et al. (1996) Diagnostic sensitivity of immunodominant epitopes of glutamic acid decarboxylase (GAD65) autoantibodies in childhood IDDM. Diabetologia 39: 1091-1098

34. Tuomi T, Rowley MJ, Knowles WJ et al. (1994) Autoantigenic properties of native and denatured glutamic acid decarboxylase: evidence for a conformational epitope. Clin Immunol Immunopathol 71: 53-59

35. Kim J, Namchuk M, Bugawan T et al. (1994) Higher autoantibody levels and recognition of a linear NH2-terminal epitope in the autoantigen GAD65 distinguish Stiff-ManSyndrome from insulin-dependent diabetes mellitus. J Exp Med 180: 595-606

36. Parish NM, Hutchings PR, O'Reilly L et al. (1995) Tolerance induction as a therapeutic strategy for the control of autoimmune endocrine disease in mouse models. Immunol Rev 144: 269-300 